\title{
Cauchy-Neumann Problem for Second-Order General Schrödinger Equations in Cylinders with Nonsmooth Bases
}

\author{
Nguyen Manh Hung, ${ }^{1}$ Tran Xuan Tiep, ${ }^{2}$ \\ and Nguyen Thi Kim Son ${ }^{1}$ \\ ${ }^{1}$ Department of Mathematics, Hanoi University of Education, Hanoi, Vietnam \\ ${ }^{2}$ Faculty of Applied Mathematics and Informatics, Hanoi University of Technology, Hanoi, Vietnam \\ Correspondence should be addressed to Nguyen Thi Kim Son, mt02_02@yahoo.com
}

Received 26 February 2009; Accepted 18 June 2009

Recommended by Gary Lieberman

The main goal of this paper is to obtain the regularity of weak solutions of Cauchy-Neumann problems for the second-order general Schrödinger equations in domains with conical points on the boundary of the bases.

Copyright (c) 2009 Nguyen Manh Hung et al. This is an open access article distributed under the Creative Commons Attribution License, which permits unrestricted use, distribution, and reproduction in any medium, provided the original work is properly cited.

\section{Introduction and Notations}

Cauchy-Dirichlet problem for general Schrödinger systems in domains containing conical points has been investigated in [1,2]. Cauchy-Neumann problems have been dealt with for hyperbolic systems in [3] and for parabolic equations in [4-6]. In this paper we consider the Cauchy-Neumann problem for the second-order general Schrödinger equations in infinite cylinders with nonsmooth bases. The solvability of this problem has been considered in [7]. Our main purpose here is to study the regularity of weak solution of the mentioned problem.

The paper consists of six sections. In Section 1, we introduce some notations and functional spaces used throughout the text. A weak solution of the problem is defined in Section 2 together with some results of its unique existence and smoothness with the time variable. Our main result, the regularity with respect to both of time and spatial variables of the weak solution of the problem, is stated in Section 3. The proof of this result is given in Section 4 with some auxiliary lemmas. In Section 5 we specify that result for the classical Schrödinger equations in quantum mechanics. Finally, some conclusions of our results are given in Section 6. 
Let $\Omega$ be a bounded domain in $\mathbb{R}^{n}, n \geqslant 2 ; \bar{\Omega}$ and $\partial \Omega$ denote the closure and the boundary of $\Omega$ in $\mathbb{R}^{n}$. We suppose that $\Gamma=\partial \Omega \backslash\{0\}$ is an infinitely differentiable surface everywhere except the coordinate origin and $\Omega$ coincides with the cone $K=\{x: \quad x /|x| \in G\}$ in a neighborhood of the origin point 0 , where $G$ is a smooth domain on the unit sphere $S^{n-1}$ in $\mathbb{R}^{n}$. We begin by introducing some notations and functional spaces which are used fluently in the rest.

Denote $Q_{\infty}=\Omega \times(0,+\infty), \bar{Q}_{\infty}$ is the closure of $Q_{\infty}, S_{\infty}=\Gamma \times(0,+\infty), x=\left(x_{1}, \ldots, x_{n}\right) \in$ $\Omega, \partial_{x_{j}}=\partial / \partial x_{j}, u_{x_{j}}=\partial_{x_{j}} u, u_{t^{k}}=\partial^{k} u / \partial t^{k}, r=|x|=\sqrt{x_{1}^{2}+\cdots+x_{n}^{2}}$. For each multi-index $\alpha=$ $\left(\alpha_{1}, \ldots, \alpha_{n}\right)\left(\alpha_{i} \in \mathbb{N}, i=1, \ldots, n\right)$, set $|\alpha|=\alpha_{1}+\cdots+\alpha_{n}, \partial^{\alpha}=\partial_{x}^{\alpha}=\partial_{x_{1}}^{\alpha_{1}} \cdots \partial_{x_{n}}^{\alpha_{n}}$.

In this paper we will use usual functional spaces: $C^{\infty}(\Omega), L_{2}(\Omega), H^{m}(\Omega)$, where $m \in \mathbb{N}$ (see $[1,2]$ for the precise definitions).

Denote $H_{\beta}^{l}(\Omega)$ is a space of all measurable complex functions $u(x, t)$ that satisfy

$$
\|u\|_{H_{\beta}^{l}(\Omega)}=\left(\sum_{|\alpha| \leqslant l} \int_{\Omega} r^{2(\beta+|\alpha|-l)}\left|\partial^{\alpha} u\right|^{2} d x\right)^{1 / 2}<+\infty .
$$

$H^{m, l}\left(e^{-\gamma t}, Q_{\infty}\right)(\gamma>0)$ - a space of all measurable complex functions $u(x, t)$ that have generalized derivatives up to order $m$ with respect to $x$ and up to order $l$ with respect to $t$ with the norm

$$
\|u\|_{H^{m, l}\left(e^{-\gamma t}, Q_{\infty}\right)}=\left(\int_{Q_{\infty}}\left[\sum_{|\alpha| \leqslant m}\left|\partial^{\alpha} u\right|^{2}+\sum_{j=1}^{l}\left|u_{t}\right|^{2}\right] e^{-2 \gamma t} d x d t\right)^{1 / 2}<+\infty .
$$

$H_{\beta}^{l, k}\left(e^{-\gamma t}, Q_{\infty}\right)$-a space of all measurable complex functions $u(x, t)$ with the norm

$$
\|u\|_{H_{\beta}^{l, k}\left(e^{-\gamma t}, Q_{\infty}\right)}=\left(\int_{Q_{\infty}}\left[\sum_{|\alpha| \leqslant l} r^{2(\beta+|\alpha|-l)}\left|\partial^{\alpha} u\right|^{2}+\sum_{j=1}^{k}\left|u_{t}\right|^{2}\right] e^{-2 \gamma t} d x d t\right)^{1 / 2}<+\infty .
$$

$H_{\beta}^{l}\left(e^{-\gamma t}, Q_{\infty}\right)$ - a weighted space with the norm

$$
\|u\|_{H_{\beta}^{l}\left(e^{\left.-\gamma^{t}, Q_{\infty}\right)}\right.}=\left(\sum_{|\alpha|+j \leqslant l} \int_{Q_{\infty}} r^{2(\beta+|\alpha|+j-l)}\left|\partial^{\alpha} u_{t^{j}}\right|^{2} e^{-2 \gamma t} d x d t\right)^{1 / 2}<+\infty
$$

Let $X$ be a Banach space. Denote by $L^{\infty}(0, \infty ; X)$ a space of all measurable functions $u$ : $(0,+\infty) \rightarrow X, t \mapsto u(t)$ with the norm

$$
\|u\|_{L^{\infty}(0, \infty ; X)}=\operatorname{ess} \sup _{0<t<+\infty}\|u(t)\|_{X}<+\infty
$$




\section{Formulation of the Problem and Obvious Results}

In this paper we consider following problem:

$$
\begin{gathered}
i L u-u_{t}=f \quad \text { in } Q_{\infty}, \\
u(x, 0)=0 \quad \text { on } \Omega, \\
N u=0 \quad \text { on } S_{\infty},
\end{gathered}
$$

where $L$ is a formal self-adjoint differential operator of second-order defined in $Q_{\infty}$ :

$$
L u=L(x, t, \partial) u=\sum_{j, k=1}^{n} \frac{\partial}{\partial x_{j}}\left(a_{j k}(x, t) \frac{\partial u}{\partial x_{k}}\right)+a(x, t) u,
$$

$\left(a_{j k}(x, t)=\bar{a}_{k j}(x, t)\right.$ for all $j, k=1,2, \ldots, n ; a(x, t)=\bar{a}(x, t)$, for all $\left.(x, t) \in Q_{\infty}\right)$, and

$$
N u=N(x, t, \partial) u=\sum_{j, k=1}^{n} a_{j k}(x, t) \frac{\partial u}{\partial x_{k}} \cos \left(x_{j}, v\right)
$$

is the conormal derivative on $S_{\infty}, v$ is the unit exterior normal to $S_{\infty}, f$ is a given function.

Set

$$
B(t, u, v)=\int_{\Omega}\left(\sum_{j, k=1}^{n} a_{j k}(x, t) \frac{\partial u}{\partial x_{k}} \frac{\overline{\partial v}}{\partial x_{j}}-a(x, t) u \bar{v}\right) d x
$$

Throughout this paper, we assume that the coefficients of $L$ are infinitely differentiable and bounded in $\bar{Q}_{\infty}$ together with all their derivatives. Moreover, suppose that $a_{j k}$ are continuous in $x \in \bar{\Omega}$ uniformly with respect to $t \in(0,+\infty)$ for all $j, k=1, \ldots, n$. In addition, assume that $B(t, \cdot, \cdot)$ is $H^{1}(\Omega)$-coercive uniformly with respect to $t \in(0,+\infty)$, that is,

$$
B(t, u, u) \geqslant \mu_{0}\|u\|_{H^{1}(\Omega)}^{2} \quad \forall u \in H^{1}(\Omega), t \in(0,+\infty),
$$

where $\mu_{0}$ is a positive constant independent of $u$ and $t$.

The function $u(x, t)$ is called a weak solution in the space $H^{1,0}\left(e^{-\gamma t}, Q_{\infty}\right)$ of the problem (2.1)-(2.3) if $u(x, t) \in H^{1,0}\left(e^{-\gamma t}, Q_{\infty}\right)$, satisfying for each $T \in(0,+\infty)$

$$
\sum_{j, k=1}^{n} \int_{Q_{\infty}} a_{j k} \frac{\partial u}{\partial x_{k}} \frac{\overline{\partial \eta}}{\partial x_{j}} d x d t-\int_{Q_{\infty}} a u \bar{\eta} d x d t+i \int_{Q_{\infty}} u \overline{\eta_{t}} d x d t=i \int_{Q_{\infty}} f \bar{\eta} d x d t
$$

for all test functions $\eta(x, t) \in H^{1,1}\left(e^{-\gamma t}, Q_{\infty}\right), \eta(x, t)=0$ for all $t \in[T,+\infty)$.

Now we derive here some our obvious results of the unique existence and smoothness with respect to time variable of the weak solution of the problem (2.1)-(2.3) as lemmas of main results. 
Lemma 2.1. The solvability of the problem, (see [7, Theorems 3.1, 3.2]). There exists a positive number $\gamma_{0}$ such that if $f, f_{t} \in L^{\infty}\left(0, \infty, L_{2}(\Omega)\right)$ then for every $\gamma>\gamma_{0}$, the Cauchy-Neumann problem (2.1)(2.3) has exactly one weak solution $u(x, t)$ in $H^{1,0}\left(e^{-\gamma t}, Q_{\infty}\right)$, that satisfies

$$
\|u\|_{H^{1,0}\left(e^{-\gamma t}, Q_{\infty}\right)}^{2} \leqslant C\left(\|f\|_{L^{\infty}\left(0, \infty, L_{2}(\Omega)\right)}^{2}+\left\|f_{t}\right\|_{L^{\infty}\left(0, \infty, L_{2}(\Omega)\right)}^{2}\right)
$$

where the constant $C$ does not depend on $u, f$.

The constant $\gamma_{0}$ depends only on the operator $L$ and the dimension of the space $n$.

Lemma 2.2. The regularity with respect to time variable of the weak solution (see [7, Theorem 4.1]). Let $h$ be a nonnegative integer. Suppose that $f_{t^{k}} \in L^{\infty}\left(0, \infty, L_{2}(\Omega)\right)$ for all $k \leqslant h+1, f(x, 0)=0$ and if $h \geqslant 2$ then $f_{t^{k}}(x, 0)=0$ for all $k \leqslant h-1$, for all $x \in \Omega$. Then for every $\gamma>(2 h+1) \gamma_{0}$, the weak solution $u(x, t)$ of the problem (2.1)-(2.3) has generalized derivatives with respect to time variable up to order $h$, which belong to $H^{1,0}\left(e^{-\gamma t}, Q_{\infty}\right)$, moreover

$$
\left\|u_{t^{s}}\right\|_{H^{1,0}\left(e^{-\gamma t}, Q_{\infty}\right)}^{2} \leqslant C \sum_{k=0}^{h+1}\left\|f_{t^{k}}\right\|_{L^{\infty}\left(0, \infty, L_{2}(\Omega)\right)^{\prime}}^{2}, \quad \forall s=0,1, \ldots, h,
$$

where $C$ is a constant independent of $u, f$.

\section{Formulation of the Main Result}

Let $L_{0}(x, t, \partial)$ be the principal homogenous part of $L(x, t, \partial)$. We can write $L_{0}(0, t, \partial)$ in the form

$$
L_{0}(0, t, \partial)=r^{-2} \perp\left(\omega, t, \partial_{\omega}, r \partial_{r}\right)
$$

where $r=|x|, \omega=\left(\omega_{1}, \ldots, \omega_{n-1}\right)$ is an arbitrary local coordinate system on $S^{n-1}, \mathcal{L}$ is a linear operator with smooth coefficients.

Denote $\lambda(t)$ is an eigenvalue of Neumann problem for following equation:

$$
\mathcal{L}\left(\omega, t, \lambda(t), \partial_{\omega}\right) v(\omega)=0, \quad \omega \in G
$$

It is well known in [8] that for each $t \in(0,+\infty)$, the spectrum of this problem is an enumerable set of eigenvalues.

Recall that $\gamma_{0}$ is the positive real number in Lemma 2.1. Now, let us give the main result of the present paper.

Theorem 3.1. Let $l$ be a nonnegative integer. Assume that $u(x, t)$ is a weak solution in the space $H^{1,0}\left(e^{-\gamma t}, Q_{\infty}\right)$ with $\gamma>(2 l+5) \gamma_{0}$ of the problem $(2.1)-(2.3)$ and $f_{t^{k}} \in L^{\infty}\left(0, \infty, H_{0}^{l}(\Omega)\right)$ if $k \leqslant 3$, $f_{t^{k}}(x, 0)=0$ if $k \leqslant l+1$. In addition, suppose that in the strip

$$
1-\varepsilon-\frac{n}{2} \leqslant \operatorname{Im} \lambda \leqslant l+2-\frac{n}{2}
$$


where $\varepsilon>0$ or $\varepsilon=0$ according to $n=2$ or $n>2$, there is no point from the spectrum of the Neumann problem for the equation (3.2) for all $t \in(0,+\infty)$. Then we have $u \in H_{0}^{l+2}\left(e^{-\gamma t}, Q_{\infty}\right)$ and the following estimate holds

$$
\|u\|_{H_{0}^{l+2}\left(e^{-\gamma t}, Q_{\infty}\right)}^{2} \leqslant C \sum_{k=0}^{3}\left\|f_{t^{k}}\right\|_{L^{\infty}\left(0, \infty, H_{0}^{l}(\Omega)\right)^{\prime}}^{2}
$$

where $C$ is a constant independent of $u, f$.

\section{Proof of Theorem 3.1}

By using the same arguments as in $[1,2]$ and Lemmas 2.1, 2.2, we can prove following lemma.

Lemma 4.1. Let $\gamma>3 \gamma_{0}$ arbitrary. Assume that $u(x, t)$ is a weak solution of the problem (2.1)(2.3) in the space $H^{1,0}\left(e^{-\gamma t}, Q_{\infty}\right)$ and $f, f_{t}, f_{t t} \in L^{\infty}\left(0, \infty, L_{2}(\Omega)\right), f(x, 0)=0$. Then for almost all $t \in(0,+\infty)$ the equation

$$
\sum_{k, j=1}^{n} \int_{\Omega} a_{j k} \frac{\partial u}{\partial x_{k}} \overline{\frac{\partial X}{\partial x_{j}}} d x-\int_{\Omega} a u \bar{X} d x=i \int_{\Omega}\left[u_{t}+f\right] \bar{X} d x
$$

holds for all functions $X=X(x) \in H^{1}(\Omega)$.

Now we surround the origin by a neighborhood $U_{0}$ with a sufficiently small diameter such that the intersection of $\Omega$ and $U_{0}$ coincides with the cone $K$. We begin by proving some auxiliary lemmas.

Lemma 4.2. Let $u(x, t)$ be a weak solution in $H^{1,0}\left(e^{-\gamma t}, Q_{\infty}\right)\left(\gamma>3 \gamma_{0}\right)$ of the problem (2.1)-(2.3) such that $u(x, t)=0$ outside $U_{0}$. Moreover, we assume that $f, f_{t}, f_{t t} \in L^{\infty}\left(0, \infty, L_{2}(\Omega)\right), f(x, 0)=0$. Then for almost all $t \in(0,+\infty)$, one has

(i) if $n \geqslant 3$ then $u \in H_{1}^{2}(\Omega)$,

(ii) if $n=2$ then $u \in H_{1+\varepsilon}^{2}(\Omega)$, where $\varepsilon>0$ arbitrary.

Proof. Because $f, f_{t}, f_{t t} \in L^{\infty}\left(0, \infty, L_{2}(\Omega)\right), f(x, 0)=0$, from Lemma 2.2 we have $u_{t} \in$ $H^{1,0}\left(e^{-\gamma t}, Q_{\infty}\right)$ or $u_{t} \in L_{2}(\Omega)$ for almost all $t \in(0,+\infty)$. Following Lemma 4.1, $u(x, t)$ is a solution of the Neumann problem for elliptic equation

$$
L u=F,
$$

where $F=-i\left(u_{t}+f\right) \in L_{2}(\Omega)$ for almost all $t \in(0,+\infty)$.Denote $\Omega^{k}=\left\{x \in \Omega: 2^{-k} \leqslant|x| \leqslant\right.$ $\left.2^{-k+1}\right\}, k=1,2, \ldots$. Let $k_{0}$ be large enough such that $\Omega_{k_{0}-1} \subset U_{0}$. By choosing a smooth domain $\Omega_{k_{0}}^{\prime}$ such that $\Omega_{k_{0}} \subset \Omega_{k_{0}}^{\prime} \subset \Omega_{k_{0}-1} \cup \Omega_{k_{0}} \cup \Omega_{k_{0}+1}$, from the theory of the regular of solutions of the boundary value problem for elliptic systems in smooth domains and near the piece smooth 
boundary of domain (see [9] for reference), we have $u \in H^{2}\left(\Omega_{k_{0}}^{\prime}\right)$ for almost all $t \in(0,+\infty)$ and the following inequality holds

$$
\|\left. u(x, t)\right|_{H^{2}\left(\Omega_{k_{0}}^{\prime}\right)} ^{2} \leqslant C\left[\|F(x, t)\|_{L_{2}\left(\Omega_{k_{0}}^{\prime}\right)}^{2}\|+\| u(x, t) \|_{L_{2}\left(\Omega_{k_{0}}^{\prime}\right)}^{2}\right],
$$

where $C$ is a positive constant independent of $u, F$. It follows

$$
\int_{\Omega_{k_{0}}}\left|\partial^{\alpha} u(x, t)\right|^{2} d x \leqslant C \int_{\Omega_{k_{0}-1} \cup \Omega_{k_{0}} \cup \Omega_{k_{0}+1}}\left[|F(x, t)|^{2}+|u(x, t)|^{2}\right] d x, \quad \forall|\alpha| \leqslant 2 .
$$

By choosing $k_{1}>k_{0}$ and setting $x=\left(2^{k_{0}} / 2^{k_{1}}\right) x^{\prime}$, one has

$$
\int_{\Omega_{k_{0}}}\left|\partial^{\alpha} u\left(x^{\prime}, t\right)\right|^{2} d x^{\prime} \leqslant C \int_{\Omega_{k_{0}-1} \cup \Omega_{k_{0}} \cup \Omega_{k_{0}+1}}\left[\left|F\left(x^{\prime}, t\right)\right|^{2}\left(\frac{2^{k_{0}}}{2^{k_{1}}}\right)^{4}+\left|u\left(x^{\prime}, t\right)\right|^{2}\right] d x^{\prime}, \quad|\alpha| \leqslant 2 .
$$

Return to the variable $x$, we get

$$
\left(\frac{2^{k_{0}}}{2^{k_{1}}}\right)^{2|\alpha|} \int_{\Omega_{k_{1}}}\left|\partial^{\alpha} u(x, t)\right|^{2} d x \leqslant C \int_{\Omega_{k_{1}-1} \cup \Omega_{k_{1}} \cup \Omega_{k_{1}+1}}\left[|F(x, t)|^{2}\left(\frac{2^{k_{0}}}{2^{k_{1}}}\right)^{4}+|u(x, t)|^{2}\right] d x,
$$

where the positive constant $C$ is independent of $u, f, k_{1}$.

Case $1(n \geqslant 3)$. Then

$$
\int_{\Omega} r^{-2}|u|^{2} d x \leqslant C \int_{\Omega} r^{-n}|u|^{2} d x<+\infty
$$

It follows from (4.6) that

$$
\int_{\Omega_{k_{1}}} r^{2(|\alpha|-1)}\left|\partial^{\alpha} u\right|^{2} d x \leqslant C \int_{\Omega_{k_{1}-1} \cup \Omega_{k_{1}} \cup \Omega_{k_{1}+1}}\left[|F|^{2} r^{2}+r^{-2}|u|^{2}\right] d x
$$

where $C$ does not depend on $k_{1}$. Taking sum with respect to $k_{1}>k_{0}$, one has

$$
\sum_{k_{1}>k_{0}} \int_{\Omega_{k_{1}}} r^{2(|\alpha|-1)}\left|\partial^{\alpha} u\right|^{2} d x \leqslant C \sum_{k_{1} \geqslant k_{0}} \int_{\Omega_{k_{1}}}\left[|F|^{2} r^{2}+r^{-2}|u|^{2}\right] d x
$$

This implies

$$
\int_{\bigcup_{k>k_{0}} \Omega_{k}} r^{2(|\alpha|-1)}\left|\partial^{\alpha} u\right|^{2} d x \leqslant C \int_{\bigcup_{k} \geqslant k_{0}} \Omega_{k}\left[|F|^{2} r^{2}+r^{-2}|u|^{2}\right] d x
$$


Because in out of a neighborhood of conical point $\Omega$ is a smooth domain, so we have

$$
\int_{\Omega} r^{2(|\alpha|-1)}\left|\partial^{\alpha} u\right|^{2} d x \leqslant C \int_{\Omega}\left[|F|^{2} r^{2}+r^{-2}|u|^{2}\right] d x
$$

for all $|\alpha| \leqslant 2$, almost all $t \in(0,+\infty)$. From (4.7), (4.11) and $F \in L_{2}(\Omega)$ we receive $u \in H_{1}^{2}(\Omega)$ for almost all $t \in(0,+\infty)$.

Case $2(n=2)$. Since $u \in H^{1,0}\left(e^{-\gamma t}, Q_{\infty}\right)$ so for almost all $t \in(0,+\infty)$ one has $\int_{\Omega} r^{0}\left|\partial^{\beta} u\right|^{2} d x<$ $+\infty,|\beta|=1$. This implies $\int_{K} r^{2 \varepsilon}\left|\partial^{\beta} u\right|^{2} d x \leqslant C \int_{K}\left|\partial^{\beta} u\right|^{2} d x<+\infty$, where $\varepsilon>0$ arbitrary, $C$ is a positive constant. Because $u \equiv 0$ outside $U_{0}$, so we have

$$
\int_{\Omega} r^{2 \varepsilon}\left|\partial^{\beta} u\right|^{2} d x \leqslant C \int_{\Omega}\left|\partial^{\beta} u\right|^{2} d x<+\infty
$$

For all $\varepsilon>0$ we have $2 \varepsilon>0=1-n / 2$, so it follows from [8, Lemma 7.1.1, page 268] that

$$
\int_{\Omega} r^{2(\varepsilon-1)}|u|^{2} d x \leqslant C \sum_{|\beta|=1} \int_{\Omega} r^{2 \varepsilon}\left|\partial^{\beta} u\right|^{2} d x \leqslant C \sum_{|\beta|=1} \int_{\Omega}\left|\partial^{\beta} u\right|^{2} d x<+\infty
$$

From the inequality (4.6), for all $|\alpha| \leqslant 2$ one gets

$$
\int_{\Omega_{k_{1}}} r^{2(|\alpha|-1+\varepsilon)}\left|\partial^{\alpha} u\right|^{2} d x \leqslant C \int_{\Omega_{k_{1}-1} \cup \Omega_{k_{1}} \cup \Omega_{k_{1}+1}}\left[|F|^{2} r^{2(\varepsilon+1)}+r^{2(\varepsilon-1)}|u|^{2}\right] d x,
$$

where $C$ does not depend on $u, f, k_{1}$. By using analogous arguments used in Case 1 , from (4.13), (4.14) we have

$$
\int_{\Omega} r^{2(1+\varepsilon+|\alpha|-2)}\left|\partial^{\alpha} u\right|^{2} d x \leqslant C \int_{\Omega}\left[|F|^{2}+\sum_{|\beta|=1}\left|\partial^{\beta} u\right|^{2}\right] d x<+\infty,
$$

for all $|\alpha| \leqslant 2$, almost all $t \in(0,+\infty)$. That is $u \in H_{1+\varepsilon}^{2}(\Omega)$. The lemma is proved.

Lemma 4.3. Let $f_{t^{k}} \in L^{\infty}\left(0, \infty, L_{2}(\Omega)\right), k \leqslant 3$, and $f(x, 0)=f_{t}(x, 0)=0$ for $x \in \Omega$. Assume that $u(x, t)$ is a weak solution in $H^{1,0}\left(e^{-\gamma t}, Q_{\infty}\right)\left(\gamma>5 \gamma_{0}\right)$ of the problem $(2.1)-(2.3)$ such that $u \equiv 0$ outside $U_{0}$. In addition, suppose that the strip

$$
1-\varepsilon-\frac{n}{2} \leqslant \operatorname{Im} \lambda \leqslant 2-\frac{n}{2}
$$

where $\varepsilon=0$ or $\varepsilon>0$ according to $n \geqslant 3$ or $n=2$, does not contain any point of the spectrum of the Neumann problem for the equation (3.2) for all $t \in(0,+\infty)$. Then $u \in H_{0}^{2}\left(e^{-\gamma t}, Q_{\infty}\right)$. 
Proof. We can rewrite (2.1) in the form

$$
L_{0}(0, t, \partial) u=\widehat{F}(x, t)=-i\left(u_{t}+f\right)+\left[L_{0}(0, t, \partial)-L(x, t, \partial)\right] u
$$

If $n \geqslant 3$ then by applying Lemma 4.2 we have $u \in H_{1}^{2}(\Omega)$. In another way, because $a_{j k}$ are continuous in $x \in \bar{\Omega}$ uniformly with respect to $t \in(0,+\infty)$ for all $j, k=1, \ldots, n$ then $\left|a_{j k}(x, t)-a_{j k}(0, t)\right| \leqslant C|x|$, for all $t \in(0,+\infty)$ and $C$ is a constant independent of $t$. Therefore, from the hypotheses of this lemma one gets $\widehat{F} \in L_{2}(\Omega)$ for almost all $t \in(0,+\infty)$. Since in the strip $1-n / 2 \leqslant \operatorname{Im} \lambda \leqslant 2-n / 2$ there is no spectral point of the Neumann problem for the equation (3.2) for all $t \in(0,+\infty)$, then following results of the work [9], one gets $u \in H_{0}^{2}(\Omega)$ and satisfies

$$
\|u\|_{H_{0}^{2}(\Omega)}^{2} \leqslant C\left[\|\widehat{F}\|_{L_{2}(\Omega)}^{2}+\|u\|_{H_{1}^{2}(\Omega)}^{2}\right]
$$

for almost all $t \in(0,+\infty)$, where $C$ is a positive constant. Using the same arguments in the proof of Lemma 4.2, we have

$$
\|u\|_{H_{0}^{2}(\Omega)}^{2} \leqslant C\left(\left\|u_{t}\right\|_{L_{2}(\Omega)}^{2}+\|f\|_{L_{2}(\Omega)}^{2}+\|u\|_{H^{1}(\Omega)}^{2}\right)
$$

for almost all $t \in(0,+\infty)$. Multiplying this inequality with $e^{-2 \gamma t}$, then integrating with respect to $t$ from 0 to $+\infty$, from Lemma 2.2 one gets

$$
\|u\|_{H_{0}^{2,0}\left(e^{-\gamma t}, Q_{\infty}\right)}^{2} \leqslant C \sum_{k=0}^{3}\left\|f_{t^{k}}\right\|_{L^{\infty}\left(0, \infty, L_{2}(\Omega)\right)}^{2}<+\infty
$$

Then $u$ is a function in the space $H_{0}^{2,0}\left(e^{-\gamma t}, Q_{\infty}\right)$.

If $n=2$ then following Lemma 4.2 we have $u \in H_{1+\varepsilon}^{2}(\Omega)$ for almost all $t \in(0,+\infty)$. This and the property of the functions $a_{j k}$ continuous in $x \in \bar{\Omega}$ uniformly with respect to $t \in(0,+\infty)$ follows $\widehat{F} \in H_{1}^{0}(\Omega)$. Because the strip $1-\varepsilon-n / 2 \leqslant \operatorname{Im} \lambda \leqslant 1-n / 2$ does not contain any spectral point of the Neumann problem for (3.2), so from results of the work [9] we have $u \in H_{1}^{2}(\Omega)$ satisfying

$$
\|u\|_{H_{1}^{2}(\Omega)}^{2} \leqslant C\left[\|\widehat{F}\|_{H_{1}^{0}(\Omega)}^{2}+\|u\|_{H_{1+\varepsilon}^{2}}^{2}\right]
$$

Repeating the proof in the case $n \geqslant 3$ we achieve $u \in H_{0}^{2,0}\left(e^{-\gamma t}, Q_{\infty}\right)$, too.

Now differentiating (2.1) with respect to $t$, we have

$$
L v=F_{1}=-i\left(v_{t}+f_{t}\right)+L_{t} u,
$$

where $v=u_{t}, L_{t}=\sum_{j, k=1}^{n}\left(\partial / \partial x_{j}\right)\left(\left(a_{j k}\right)_{t}\left(\partial / \partial x_{k}\right)\right)+(a)_{t}$. From the hypotheses of the operator $L$ and Lemma 2.2 we have $F_{1} \in L_{2}(\Omega)$ for almost all $t \in(0,+\infty)$. Repeating arguments used for function $u$ we receive $v \in H_{0}^{2,0}\left(e^{-\gamma t}, Q_{\infty}\right)$ or $u_{t} \in H_{0}^{2,0}\left(e^{-\gamma t}, Q_{\infty}\right)$. 
In another way, it follows from Lemma 2.2 that

$$
\int_{Q_{\infty}}\left|u_{t^{2}}\right|^{2} e^{-2 \gamma t} d x d t<+\infty
$$

From (4.23) and the assertion that both $u$ and $u_{t}$ are in the space $H_{0}^{2,0}\left(e^{-\gamma t}, Q_{\infty}\right)$ we have $u \in H_{0}^{2}\left(e^{-\gamma t}, Q_{\infty}\right)$. This lemma is proved.

Lemma 4.4. Let $l$ be a nonnegative integer number, $\gamma$ be a real number satisfying $\gamma>(2 l+5) \gamma_{0}$, $u(x, t)$ be a weak solution in $H^{1,0}\left(e^{-\gamma t}, Q_{\infty}\right)$ of the problem $(2.1)-(2.3)$ such that $u \equiv 0$ outside $U_{0}$. Assume that $f_{t^{k}} \in L^{\infty}\left(0, \infty, H_{0}^{l}(\Omega)\right), k \leqslant 3$, and $f_{t^{k}}(x, 0)=0$ for $k \leqslant l+1, x \in \Omega$. Moreover, suppose that the strip

$$
1-\varepsilon-\frac{n}{2} \leqslant \operatorname{Im} \lambda \leqslant l+2-\frac{n}{2}
$$

does not contain any point of the spectrum of the Neumann problem for the equation (3.2) for all $t \in(0,+\infty)$, where $\varepsilon=0$ or $\varepsilon>0$ according to $n \geqslant 3$ or $n=2$. Then $u \in H_{0}^{l+2}\left(e^{-\gamma t}, Q_{\infty}\right)$, satisfying

$$
\|u\|_{H_{0}^{l+2}\left(e^{-\gamma^{t}}, Q_{\infty}\right)}^{2} \leqslant C \sum_{k=0}^{3}\left\|f_{t^{k}}\right\|_{L^{\infty}\left(0, \infty, H_{0}^{l}(\Omega)\right)^{\prime}}^{2}
$$

where the constant $C$ is independent of $u, f$.

Proof. We use the induction by $l$. For $l=0$ then we had Lemma 4.3 with noting that $H_{0}^{0}(\Omega) \equiv$ $L_{2}(\Omega)$. Assume that lemma's assertion holds up to $l-1$, we need to prove this holds up to $l$. It means that we have to prove following inequality:

$$
\left\|u_{t^{j}}\right\|_{H_{0}^{l+2-j}\left(e^{-\gamma t}, Q_{\infty}\right)}^{2} \leqslant C \sum_{k=0}^{3}\left\|f_{t^{k}}\right\|_{L^{\infty}\left(0, \infty, H_{0}^{l}(\Omega)\right)^{\prime}}^{2}
$$

for $j=l, l-1, \ldots, 0$, where $C$ is a positive constant.

Since $f_{t^{k}} \in L^{\infty}\left(0, \infty, H_{0}^{l}(\Omega)\right)$ for $k \leqslant 3$, so $f_{t^{k}} \in L^{\infty}\left(0, \infty, L_{2}(\Omega)\right)$ for $k \leqslant l+3$. In another way, $f_{t^{k}}(x, 0)=0$ for $k \leqslant l+1$. Then from Lemma 2.2 we have $u_{t^{l+1}} \in H^{1,0}\left(e^{-\gamma t}, Q_{\infty}\right), u_{t^{s}} \in$ $\left.H^{1,0}\left(e^{-\gamma t}, Q_{\infty}\right)\right)$ for all $s \leqslant l$. Hence, by using similar arguments in the proof of Lemma 4.3 we get $u_{t^{l}} \in H_{0}^{2}\left(e^{-\gamma t}, Q_{\infty}\right)$. This means that (4.26) holds for $j=l$.

Assume that (4.26) holds for $j=l, l-1, \ldots, s+1$. By putting $v=u_{t^{s}} \in H_{0}^{l-s+1}\left(e^{-\gamma t}, Q_{\infty}\right)$ (by inductive hypothesis) and differentiating (2.1) s-times with respect to $t$, we have

$$
L v=-i\left(v_{t}+f_{t^{s}}\right)+\sum_{p=1}^{s} C_{s}^{p} L_{t^{p}} \boldsymbol{u}_{t^{s-p}},
$$

where $L_{t^{p}}=\sum_{j, k=1}^{n}\left(\partial / \partial x_{j}\right)\left(\left(a_{j k}\right)_{t^{p}}\left(\partial / \partial x_{k}\right)\right)+(a)_{t^{p}}$. Following the assumptions of the induction of $s$ and the hypotheses of the function $f$ one has $v_{t} \in H_{0}^{l-s+1}\left(e^{-\gamma t}, Q_{\infty}\right), f_{t^{s}} \in H_{0}^{l-s}\left(e^{-\gamma t}, Q_{\infty}\right)$. It follows $F_{s}=-i\left(v_{t}+f_{t^{s}}\right)+\sum_{p=1}^{s} C_{s}^{p} L_{t^{p}} \mathcal{u}_{t^{s-p}} \in H_{0}^{l-s}\left(e^{-\gamma t}, Q_{\infty}\right)$. In another way since 
$H_{0}^{l-s}\left(e^{-\gamma t}, Q_{\infty}\right) \subseteq H_{-1}^{l-s-1,0}\left(e^{-\gamma t}, Q_{\infty}\right)$, so we have $F_{s} \in H_{-1}^{l-s-1}\left(e^{-\gamma t}, Q_{\infty}\right)$ for almost all $t \in$ $(0,+\infty)$. Because the strip $l+1-s-n / 2 \leqslant \operatorname{Im} l \leqslant l+2-s-n / 2$ does not contain any point of the spectrum of the Neumann problem for (3.2) for all $t \in(0,+\infty)$, then following results of the work [9], one gets $v \in H_{-1}^{l+1-s}(\Omega)$. This implies $v \in H_{-1}^{l+1-s, 0}\left(e^{-\gamma t}, Q_{\infty}\right)$. Note that $F_{s} \in H_{0}^{l-s, 0}\left(e^{-\gamma t}, Q_{\infty}\right)$ then by applying [8, Theorem 7.3.2] one gets $v \in H_{0}^{l+2-s, 0}\left(e^{-\gamma t}, Q_{\infty}\right)$ satisfying

$$
\|v\|_{H_{0}^{l+2-s, 0}\left(e^{-\gamma t}, Q_{\infty}\right)}^{2} \leqslant C\left(\left\|F_{s}\right\|_{H_{0}^{l-s, 0}\left(e^{-\gamma t}, Q_{\infty}\right)}^{2}+\|v\|_{H_{-1}^{l+1-s, 0}\left(e^{-\gamma t}, Q_{\infty}\right)}^{2}\right)
$$

where $C$ is a positive constant. In another way, it is easy to see that

$$
\left\|u_{t^{s}}\right\|_{H_{0}^{l+2-s}\left(e^{\left.-\gamma^{t}, Q_{\infty}\right)}\right.}^{2} \leqslant\left\|u_{t^{s+1}}\right\|_{H_{0}^{l+2-s-1}\left(e^{\left.-\gamma^{t}, Q_{\infty}\right)}\right.}^{2}+\left\|u_{t^{s}}\right\|_{H_{0}^{l+2-s, 0}\left(e^{-\gamma t}, Q_{\infty}\right)}^{2} .
$$

Hence from the inductive assumptions we receive

$$
\left\|u_{t^{s}}\right\|_{H_{0}^{l+2-s}\left(e^{-\gamma t}, Q_{\infty}\right)}^{2} \leqslant C \sum_{k=0}^{3}\left\|f_{t^{k}}\right\|_{L^{\infty}\left(0, \infty, H_{0}^{l}(\Omega)\right)^{\prime}}^{2}
$$

where $C$ is a constant independent of $u, f$. It means that (4.26) is proved. Finally we only need to fix $j=0$ in (4.26) to complete the proof of this lemma.

Now let us prove Theorem 3.1.

Proof. Denote $u_{0}=\varphi_{0} u$, where $\varphi_{0} \in \stackrel{o}{C^{\infty}}\left(U_{0}\right)$ and $\varphi_{0} \equiv 1$ in a neighborhood of coordinate origin. The function $u_{0}$ satisfies

$$
i L u_{0}-\left(u_{0}\right)_{t}=\varphi_{0} f+L_{1} u
$$

where $L_{1} u$ is a linear differential operator order 1 . Coefficients of this operator depend on the choice of the function $\varphi_{0}$ and equal to 0 outside $U_{0}$. Denote $u_{1}=\varphi_{1} u=\left(1-\varphi_{0}\right) u$. It is easy to see that $u_{1}$ is equal to 0 in a neighborhood of conical point. Therefore we can apply the theorem on the smoothness of a solution of elliptic problem in a smooth domain to this function to conclude that $u_{1}=\varphi_{1} u \in H_{0}^{l+2}(\Omega)$ for almost all $t \in(0,+\infty)$. By applying Lemma 2.2 we receive $u_{1} \in H_{0}^{l+2}\left(e^{-\gamma t}, Q_{\infty}\right)$ and

$$
\left\|u_{1}\right\|_{H_{0}^{l+2}\left(e^{-\gamma t}, Q_{\infty}\right)}^{2} \leqslant C \sum_{k=0}^{3}\left\|f_{t^{k}}\right\|_{L^{\infty}\left(0, \infty, H_{0}^{l}(\Omega)\right)^{\prime}}^{2} \quad C=\text { const }>0 .
$$

Now, let us prove Theorem 3.1 by induction by $l$. When $l=0$ then functions $u_{0}, \widehat{f}=$ $\varphi_{0} f+L_{1} u$ satisfy the hypotheses of Lemma 4.3. So $u_{0} \in H_{0}^{2}\left(e^{-\gamma t}, Q_{\infty}\right)$. It follows that $u=$ $u_{0}+u_{1}$ is in $H_{0}^{2}\left(e^{-\gamma t}, Q_{\infty}\right)$. Assume that the theorem holds up to $l-1$ then we have $u \in$ $H_{0}^{l+1}\left(e^{-\gamma t}, Q_{\infty}\right)$. By using analogous arguments in the proof of Lemma 4.4, with note that $\widehat{f}_{t^{s}} \in$ $H_{0}^{l-s}\left(e^{-\gamma t}, Q_{\infty}\right)$ (from the hypothesis of induction), we can prove that $u_{0} \in H_{0}^{l+2}\left(e^{-\gamma t}, Q_{\infty}\right)$. So 
$u \in H_{0}^{l+2}\left(e^{-\gamma t}, Q_{\infty}\right)$. The inequality in Theorem 3.1 can derive from inequality (4.25) (for $u_{0}$ ) and inequality (4.32). The theorem is proved completely.

\section{Cauchy-Neumann Problem For Classical Schrödinger Equation In Quantum Mechanics}

In this section we apply the previous result to the Cauchy-Neumann problem for classical Schrödinger equations in quantum mechanics. It is shown that the smoothness of the weak solution of this problem depends on the structure of the boundary of the domain, the right hand side and the dimension $n$ of the space $\mathbb{R}^{n}$.

The classical Schrödinger equation in quantum mechanics has the form

$$
i \Delta u(x, t)-u_{t}(x, t)=f(x, t),
$$

where $\Delta$ is the Laplace operator. Now we consider the Cauchy-Neumann problem for (5.1) in infinite cylinder $Q_{\infty}$ with the initial condition

$$
u(x, 0)=0 \quad \text { on } \Omega,
$$

and the boundary condition

$$
\frac{\partial u}{\partial v}=\sum_{k=1}^{n} \frac{\partial u}{\partial x_{k}} \cos \left(x_{k}, v\right)=0 \quad \text { on } S_{\infty}
$$

where $v$ is the unit exterior normal to $S_{\infty}$.

The Laplace operator in polar coordinate $(r, \omega)$ in $\mathbb{R}^{n}$ can be written in the form

$$
\Delta u(r, \omega)=\frac{1}{r^{n-1}} \frac{\partial}{\partial r}\left(r^{n-1} \frac{\partial}{\partial r}\right) u(r, \omega)+\frac{1}{r^{2}} \Delta_{\omega} u(r, \omega),
$$

where $\Delta_{\omega}$ is the Laplace-Beltrami operator on the unit sphere $S^{n-1}$. Therefore, the corresponding spectral problem for (3.2) is the Neumann problem for following equation:

$$
\Delta_{\omega} v+\left[(i \lambda)^{2}+i(2-n) \lambda\right] v=0, \quad \omega \in G
$$

The regularity of the weak solution of the problem (5.1)-(5.3) can be stayed as follows.

Theorem 5.1. Let $n>4, u$ be a weak solution in the space $H^{1,0}\left(e^{-\gamma t}, Q_{\infty}\right)\left(\gamma>5 \gamma_{0}\right)$ of the CauchyNeumann problem (5.1)-(5.3) and $f_{t^{k}} \in L^{\infty}\left(0, \infty, L_{2}(\Omega)\right)$ if $k \leqslant 3, f(x, 0)=f_{t}(x, 0)=0$. Then $u \in H_{0}^{2}\left(e^{-\gamma t}, Q_{\infty}\right)$.

Proof. Note $k$ be nonnegative eigenvalues of the Neumann problem for equation

$$
\Delta_{\omega} v+k v=0, \quad \omega \in G
$$


Then $\lambda=i\left((2-n) / 2 \pm \sqrt{((n-2) / 2)^{2}+k}\right)$ are eigenvalues of the Neumann problem for (5.5). It is easy to see that when $n>4$ the strip

$$
1-\frac{n}{2} \leqslant \operatorname{Im} \lambda \leqslant 2-\frac{n}{2}
$$

does not contain any eigenvalue of the Neumann problem for (5.5). By applying Theorem 3.1 we have $u \in H_{0}^{2}\left(e^{-\gamma t}, Q_{\infty}\right)$. The theorem is proved.

\section{Conclusions}

The Schrödinger equation has received a great deal of attention from mathematicians, in particular because of its application to quantum mechanics and optics. It is therefore important to research boundary value problems for it. Such problems have been previously proposed and analyzed for Schrödinger equations whose coefficients are independent of the time variable and in finite cylinders $Q_{T}(T<+\infty)$ (see, e.g., [10]). In infinite cylinder $Q_{\infty}$, the first initial boundary value problem for this kind of equation with coefficients depend on both of time and spatial variables has been considered (see $[1,2])$. In this paper, for a general Schrödinger equation in infinite cylinder $Q_{\infty}$ with conical points in the boundary of base, we proved regularity property of solution of second initial boundary value problem. As a special application of these new results, we received the regularity of solution of a classical Schrödinger equation in quantum mechanics when the dimension of space $n>4$. The similar questions for the case $n \leqslant 4$ can be answered after researching the asymptotic of solution in the case the strip $1-n / 2 \leqslant \operatorname{Im} \lambda \leqslant 2+l-n / 2$ contains eigenvalues of the associated spectral problem. This is also the aim of our future research.

\section{References}

[1] N. M. Hung, "The first initial boundary value problem for Schrödinger systems in non-smooth domains," Diff. Urav., vol. 34, pp. 1546-1556, 1998 (Russian).

[2] N. M. Hung and C. T. Anh, "On the smoothness of solutions of the first initial boundary value problem for Schrödinger systems in domains with conical points," Vietnam Journal of Mathematics, vol. 33, no. 2, pp. 135-147, 2005.

[3] A. Yu. Kokotov and B. A. Plamenevskir, "On the asymptotic behavior of solutions of the Neumann problem for hyperbolic systems in domains with conical points," Algebra i Analiz, vol. 16, no. 3, pp. 56-98, 2004 (Russian), English translation in St. Petersburg Mathematical Journal, vol. 16, no. 3, pp. 477-506, 2005.

[4] E. V. Frolova, "An initial-boundary value problem with a noncoercive boundary condition in a domain with edges," Zapiski Nauchnykh Seminarov (POMI), vol. 213, no. 25, pp. 206-223, 1994.

[5] N. M. Hung and N. T. Anh, "Regularity of solutions of initial-boundary value problems for parabolic equations in domains with conical points," Journal of Differential Equations, vol. 245, no. 7, pp. 18011818, 2008.

[6] V. A. Solonnikov, "On the solvability of classical initial-boundary value problem for the heat equation in a dihedral angle," Zapiski Nauchnykh Seminarov Leningradskogo Otdeleniya Matematicheskogo Instituta, vol. 127, pp. 7-48, 1983.

[7] N. M. Hung and N. T. K. Son, "Existence and smoothness of solutions to second initial boundary value problems for Schrödinger systems in cylinders with non-smooth bases," Electronic Journal of Differential Equations, vol. 2008, no. 35, pp. 1-11, 2008.

[8] V. A. Kozlov, V. G. Maz'ya, and J. Rossmann, Elliptic Boundary Value Problems in Domains with Point Singularities, vol. 52 of Mathematical Surveys and Monographs, American Mathematical Society, Providence, RI, USA, 1997. 
[9] L. Nirenberg, "Remarks on strongly elliptic partial differential equations," Communications on Pure and Applied Mathematics, vol. 8, pp. 648-674, 1955.

[10] J. L. Lions and E. Magenes, Non-Homogeneous Boundary Value Problems and Applications, vol. 1-2, Springer, New York, NY, USA, 1972. 